\title{
Properties of Radio Source Host Galaxies
}

\author{
Wim de Vries \\ Lawrence Livermore National Laboratory, IGPP, 7000 East Avenue, Livermore, CA 94550, USA \\ Received 2002 August 21, accepted 2002 September 21
}

\begin{abstract}
A brief overview of the current radio source host galaxy state of affairs is given. All the evidence appears to point towards a scenario in which the young radio source expands through the host galaxy on timescales of $10^{5}-10^{6} \mathrm{yr}$, before it ends its life as a large scale FR II radio galaxy. The place and role of the quasars in this evolutionary picture is unclear, however, and remains an issue of debate.
\end{abstract}

Keywords: galaxies: active — galaxies: nuclei — radio continuum: galaxies

\section{Introduction}

Powerful radio galaxies and quasars have contributed and will continue to contribute significantly to our understanding of galaxy evolution and the evolving AGN phenomenon. The interaction between the radio plasma and the ambient media makes these objects laboratories for galaxy ISM and IGM studies, in the nearby and distant universe. In some respects, for example $K$ band luminosity, host galaxy properties have been found to be rather homogeneous, opening the possibility of using these sources as cosmological standard candles. Their powerful radio emission makes finding these objects among the many at high $z$ rather straightforward. With more 8-m class telescopes coming online, and with the improving (near IR) detector technology, detailed study of these objects at $z>1$ is within reach. The powerful radio source population can be divided into three size classes: 1) the ultra compact gigahertz peaked spectrum (GPS) sources, with radio sizes smaller than $1 \mathrm{kpc}$, essentially on the narrow line region (NLR) size scale; 2) compact steep spectrum (CSS) sources, with radio sizes of $\lesssim 15 \mathrm{kpc}$, i.e. of subgalactic proportions; and 3) the large scale FR II sources, where the radio structure has expanded well beyond the host galaxy confines. These sources were originally considered different objects, and their classification is based on radio spectral properties. Until recently, the place of these compact radio sources within the general population of large extended double-lobed radio sources (radio galaxies and quasars) was not known: both the hypothesis of young objects in the early stage of their existence and the alternative hypothesis of 'frustrated' objects in host galaxies with dense interstellar media confining the radio source were popular. Limited imaging data suggestive of distorted hosts in groups or poor clusters of galaxies argued for the first, but the self-absorbed synchrotron spectra together with measured radio source depolarisation and optical reddening signatures argued for the second hypothesis. Theoretical and observational work during the last couple of years has demonstrated that the youth scenario is more likely. Thus, GPS and CSS radio sources can teach us about the conditions which result in the birth of a powerful radio source, the conditions of such sources developing in dense interstellar environments, and the way in which those conditions evolve with redshift. This paper will give a brief, non-exhaustive, overview of the imaging and spectroscopic progress that has been made since the previous GPS/CSS source workshop in 1996.

\section{Why are the Hosts Important?}

There are various reasons why one wants to study the host galaxies of powerful radio sources. The most obvious reason is that one needs an (optical) identification to measure redshift. This sets the distance and absolute host galaxy luminosity for a particular radio source, quantities needed to compare these sources on the same physical scale. But there are more subtle reasons too. Radio source and stellar evolutionary timescales are quite different; a radio source has a lifespan of the order of $10^{8} \mathrm{yr}$, whereas the bulk of the stars (and therefore the galaxies themselves) are around for more than $10^{10} \mathrm{yr}$. Radio sources are therefore 'born' in mature, well-evolved stellar systems, ${ }^{1}$ and can be considered products of their environment. This in turn means that we can use the hosts to study environmental properties which may pertain to the triggering of radio activity, properties like clustering/grouping of galaxies, merger activity in the host galaxy, and the presence of gas reservoirs which may be tapped to fuel the AGN. The host galaxy does not just set the stage for the radio source to evolve in, it also interacts with the radio source as it evolves. This is especially true in the early stages of the radio source expansion, where the radio structure has not expanded beyond the host galaxy (i.e. the GPS and CSS phases). With the proper high spatial resolution instruments, like HSTs optical and near IR cameras, one can directly observe the interaction between the radio plasma and the ambient gaseous medium (cf. Figure 4). Once the radio structure has expanded beyond the host galaxy (the FR II stage), the instantaneous interaction component (e.g. shock ionised gas) fades away, leaving in some cases longer lived traces in the wake of the previous interaction. For the FR II sources with this 'aligned' emission component it is usually interpreted as either jet-induced star

\footnotetext{
${ }^{1}$ Only very few GPS/CSS galaxies are known beyond $z=1$.
} 
formation (e.g. McCarthy et al. 1987; Rees 1989) or scattered AGN light off the walls of the cleared-out radio cavity (e.g. Dey et al. 1996). Since either of these scenarios only work under specific conditions, this alignment effect in FR II sources occurs less frequently than the one found in CSS/GPS sources, where it arises as a consequence of the radio source expansion (e.g. Bicknell, Dopita, \& O'Dea 1997; de Vries et al. 1999). Studying the host galaxies therefore is essential if one wants to understand the environment of the radio source, its short and long-term effects on the host, and its associated AGN. Furthermore, on a less source specific level, the open issues of the cosmological evolution of radio sources, their possible duty cycles, and the radio source role in the formation of massive black holes in the early universe can only be settled by studying hosts. The rest of the paper is divided into two parts: optical and near IR imaging results, and spectroscopic results. The latter is touched upon briefly, since O'Dea et al. (2003) will cover the same material in more detail.

\section{Imaging}

Once interest is peaked in a particular radio source, the first step is to identify its associated host galaxy optically or in the near IR. This is rather straightforward for the very nearby galaxies, but it becomes more problematic at larger distances. First of all, the galaxies themselves become much fainter and harder to detect, and secondly, unambiguously identifying the usually weak radio core with one particular galaxy becomes increasingly difficult with increasing host galaxy surface densities and decreasing radio flux. Indeed, radio host galaxies beyond redshifts of $\sim 1$ have typical $R$-band magnitudes of 24 (cf. O'Dea et al. 1996; Snellen et al. 1996), and are therefore taxing targets for 4-m class telescopes. Figure 1, left panel, shows the optical identification for the GPS source 0008-421, a source which at $R=24.3$ long defied positive identification. This task is made slightly easier in the near IR, however, where the host galaxy with its old stellar population is putting out most of its energy. The observed host $R-K$ colour is a strong function of redshift, as can be seen in Figure 1, right panel. Around $z=1$ the $R-K$ colour can be as large as 6 magnitudes! Virtually all high- $z$ radio source identifications are therefore made in the near IR (e.g. De Breuck et al. 2002).

\subsection{High Resolution Near Infrared Imaging}

The near IR is the imaging band of choice for a few reasons. Firstly, these galaxies are brighter in the near IR, which makes them easier to study. Next, extinction is much reduced compared to the optical, whether it is foreground or intrinsic extinction. The lack of dominant emission lines in the near IR further reduces the presence of non-stellar components to the signal: the near IR effectively provides us with a clean, old-stellar-population-only, handle on the host morphology. We used HST's NICMOS on a sample of GPS, CSS, and FR II galaxies to study the hosts in detail (de Vries et al. 2000b), some of which will be repeated here. Luminosity profiles and their derived quantities - effective radius $\left(R_{\mathrm{e}}\right)$, effective surface brightness $\left(\mu_{\mathrm{e}}\right)$, mean surface brightness $(\langle\mu\rangle)$ - and their general shape are all important quantities for discriminating between various objects. Spiral galaxies usually have exponential profiles, whereas ellipticals are better fitted with de Vaucouleurs' type laws $\left(\mu(r) \propto R^{1 / n}\right)$. We can, however, fit the profile with a generalised de Vaucouleurs' law $\left(R^{1 / n}\right)$. This exponent $n$ also correlates with elliptical type (e.g. Graham et al. 1996). The $n$-distribution of the sample is plotted in Figure 2, left panel. A few things are immediately
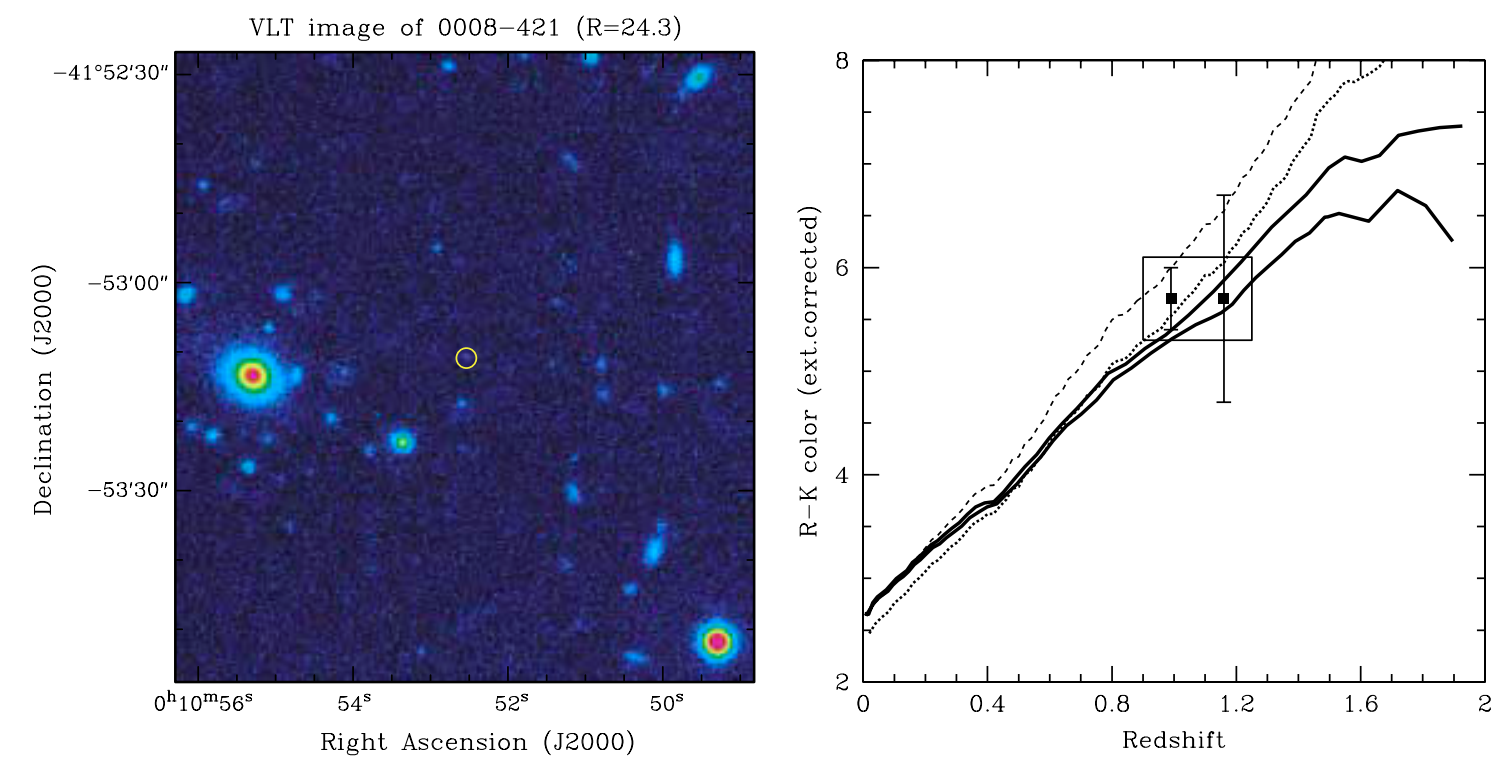

Figure 1 Left: VLT $R$-band identification image of GPS $0008-421$. Based on its $R=24.3$ magnitude, its redshift should be $z \sim 1.2$. Right: Modelled $R-K$ colours as a function of redshift. The overplotted solid tracks are passively evolving stellar populations, each with a different formation redshift. The dashed and dotted lines represent a $10 \mathrm{Gyr}$ - and 5 Gyr-old, non-evolving population (plot taken from de Vries et al. 2000a). 

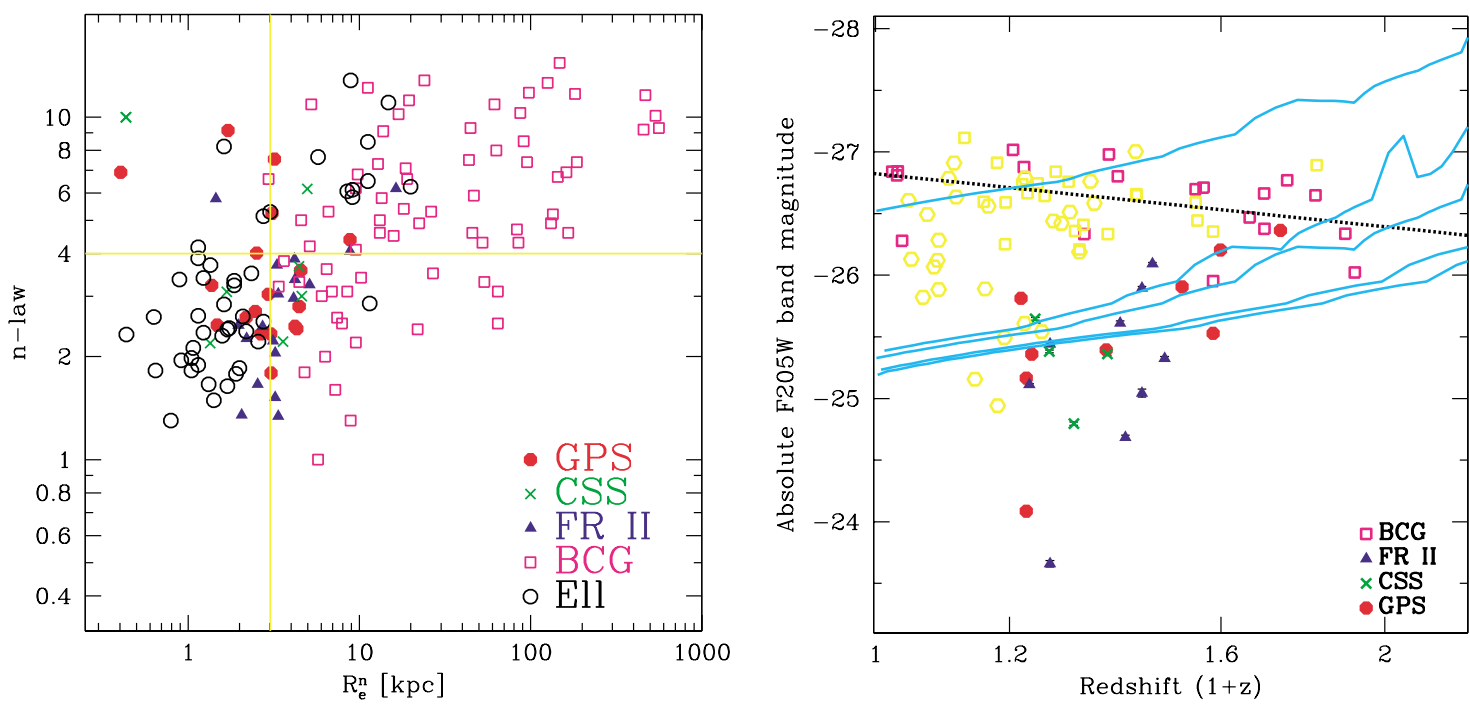

Figure 2 Left: Plot of the exponent $n$ of the $R^{1 / n}$ law versus the corresponding effective radius. Overplotted are literature data on BCGs (Graham et al. 1996) and a sample of ellipticals, ranging from small to large (Caon, Capaccioli, \& D'Onofrio 1993). The lines divide the sample into 'ordinary' and 'bright' ellipticals (lower left and upper right quadrant). Right: Absolute F205W magnitude versus redshift. The bottom set of 4 tracks are passive evolutionary tracks for $5 \times 10^{11} \mathrm{M}_{\odot}$ luminous mass, with a mean metallicity approximately solar, and formation redshifts $\left(z_{\mathrm{f}}\right)$ of $1.5,2,5$, and 8 (top to bottom), respectively. The top solid track is for $1.5 \times 10^{12} \mathrm{M}_{\odot}$ luminous mass, with $z_{\mathrm{f}}=2$. The dashed track is a best fit to the BCG sample, roughly consistent with the non-evolving case (horizontal lines in this plot). BCG data (open symbols) are taken from Aragón-Salamanca et al. (1993) and from Collins \& Mann (1998), after being converted from $K$ into F205W magnitudes. Open squares are BCGs with an X-ray luminosity in excess of $3 \times 10^{44} \mathrm{ergs} \mathrm{s}^{-1}$, open hexagons are BCGs with a lower X-ray luminosity.

clear: firstly, none of the sample objects have an exponential $(n=1)$ profile, and therefore do not seem to reside in spiral hosts. Secondly, our sample of radio galaxies overlaps neither with the $n$-value distribution for brightest cluster galaxies (BCGs), nor with the small elliptical sample. The GPS, CSS, and FR II galaxies are therefore best described as massive (giant) ellipticals, but not the most massive (BCGs). This result is corroborated by Figure 2, right panel, which plots the absolute luminosity (which correlates well with overall mass) versus redshift. Again, our hosts are less luminous than BCGs at similar redshifts. Owen \& Laing (1989) and Owen \& White (1991) find a similar result for a larger sample of classical doubles (FR IIs), which are significantly fainter than BCGs. Another striking difference is that the redshift evolution of our sample seems to be more consistent with passive evolution than that of the BCGs. The latter have to increase in luminous mass (through merging) as they evolve. Our sample, therefore, does not seem to share the same evolutionary background as the BCGs, making it less likely that GPS/CSS radio sources evolve into BCG-associated FR I sources.

\subsection{Colour Maps}

The near IR is much less affected by non-stellar emission and absorption components than the optical. So, the combination of near IR with optical imaging (a colour map) will yield direct visual evidence of features such as dust lanes, or compact regions of enhanced star formation. Two examples of the potential of these colour maps are shown in Figure 3. Basically all the sources in our NICMOS sample exhibited some signs of ongoing interaction, either in the form of close (blue) companions (like 3C 460), reddened nuclei/dust lanes, or a combination of these (de Vries et al. 2000b). The presence of these merging events is not at odds with the passive evolutionary history inferred from the profile analysis, it just indicates that we are probably witnessing the first in a series of merging events. This also does not exclude that any given source might eventually end up as a BCG (after a string of merging events), and that, if radio activity starts up again, it will become an FR I. However, it is unlikely that our sample galaxies will morph into BCGs over the typical FR II lifetime. We are therefore dealing with different epochs of radio loudness in the same host, something which does not have any implications for the evolution of an individual radio source.

\subsection{Narrowband Imaging}

As a final example, and leading up to the spectroscopy section, we present some narrowband linear ramp filter (LRF) images. These LRF images, in combination with HSTs WFPC2 camera, allow us to isolate a particular emission line (in this case [O III] $5007 \AA$ ), and to investigate its spatial structure. Figure 4 shows two examples of this technique, the sources 3C 268.3 and 3C 303.1. It is striking especially in the latter case how different the spatial distributions of the continuum and the line emitting gas are. Comparing the line emission distribution with the radio structures, however, showed a remarkable correlation between the two (de Vries et al. 1999). This strongly suggests that the interaction of the radio source with the 

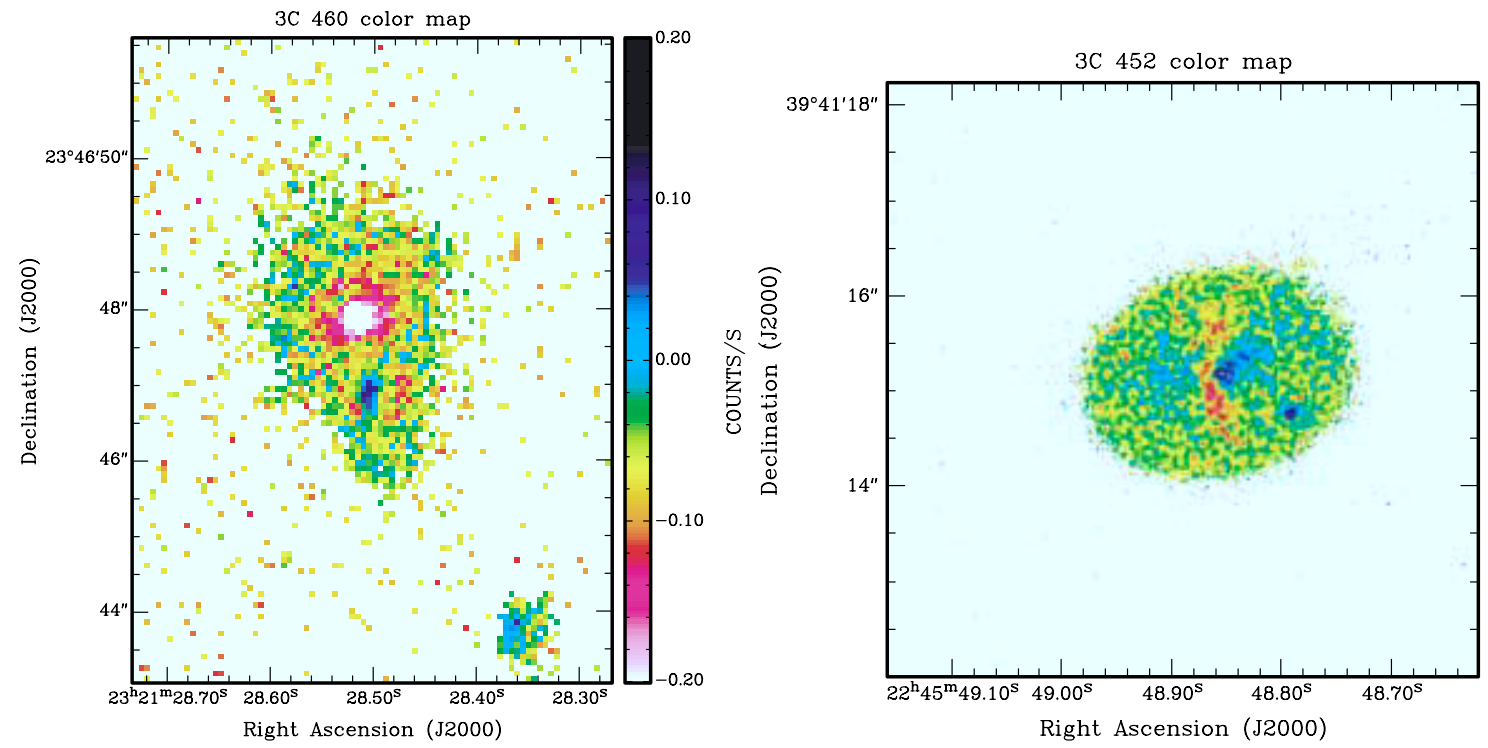

Figure 3 Left: Optical-near IR colour map of 3C 460. This is an interacting system where there are indications of enhanced star-formation rates, at least in the (smaller) merging companion. The main galaxy, however, has a rather red patch of excess emission, not centred on the nucleus, perhaps a site of intrinsic obscuration. The radio axis is pointing towards the general direction of the companion. Right: An even higher spatial resolution colour map, using HST in the optical and Keck+AO in the near IR. The source is $3 \mathrm{C} 452$, which for the first time sports its dust torus and a small blue companion.
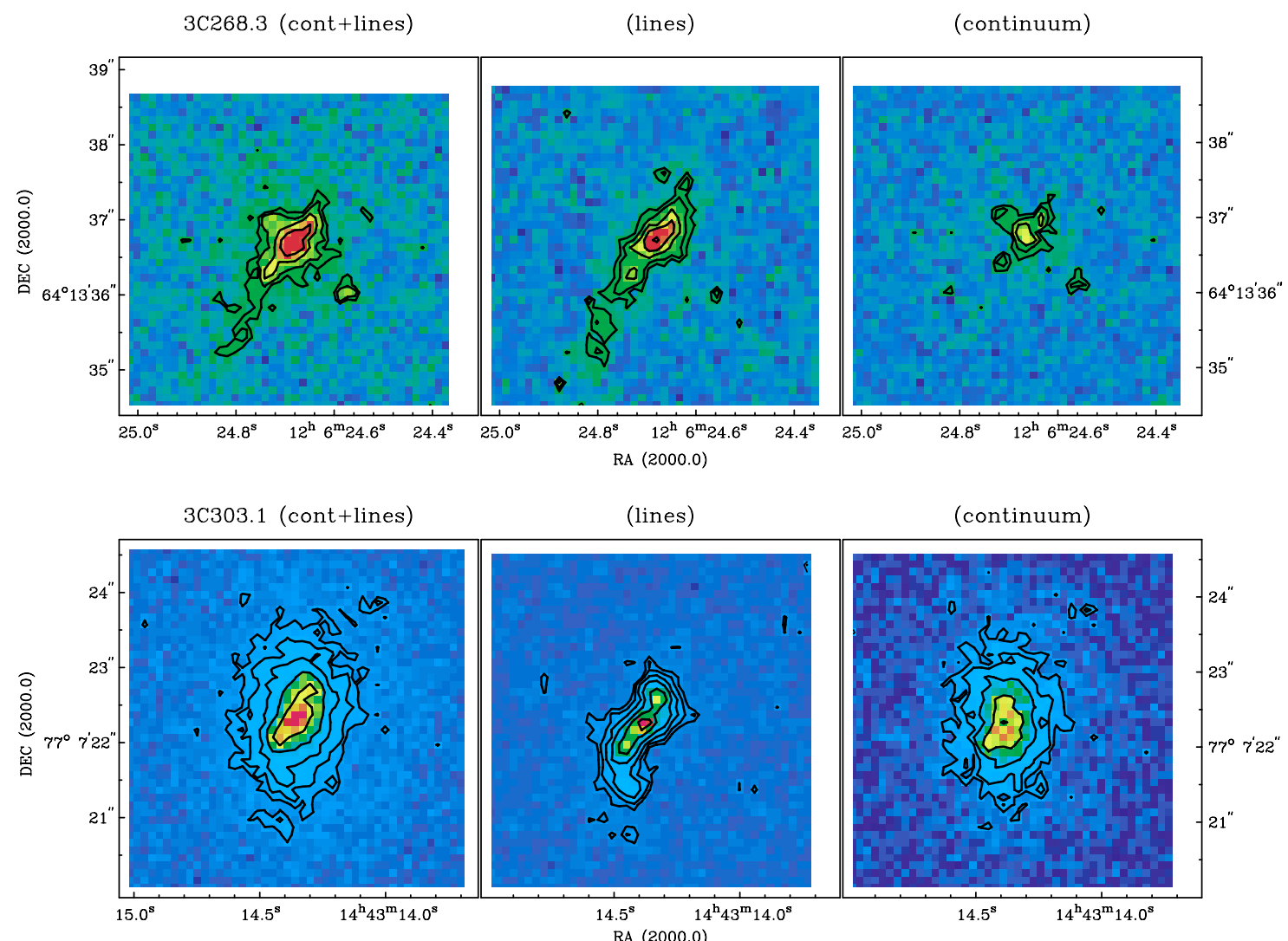

Figure 4 Linear ramp filter images of 3C 268.3 and 3C 303.1, centred on the [O III] 5007 $\AA$ emission line. Left panel displays the broadband F702W image, and the middle and right panels are pure line emission and pure continuum images, respectively.

ambient gas is responsible for the morphology and possibly the energetics of the emission line gas, something we followed up using STIS (cf. Section 4; O'Dea et al. 2003).

\section{Spectroscopy}

Spectroscopy is an important tool, not just for redshift measurements, but also for detailed investigations on the nature of the line emission regions of the sources. Line 

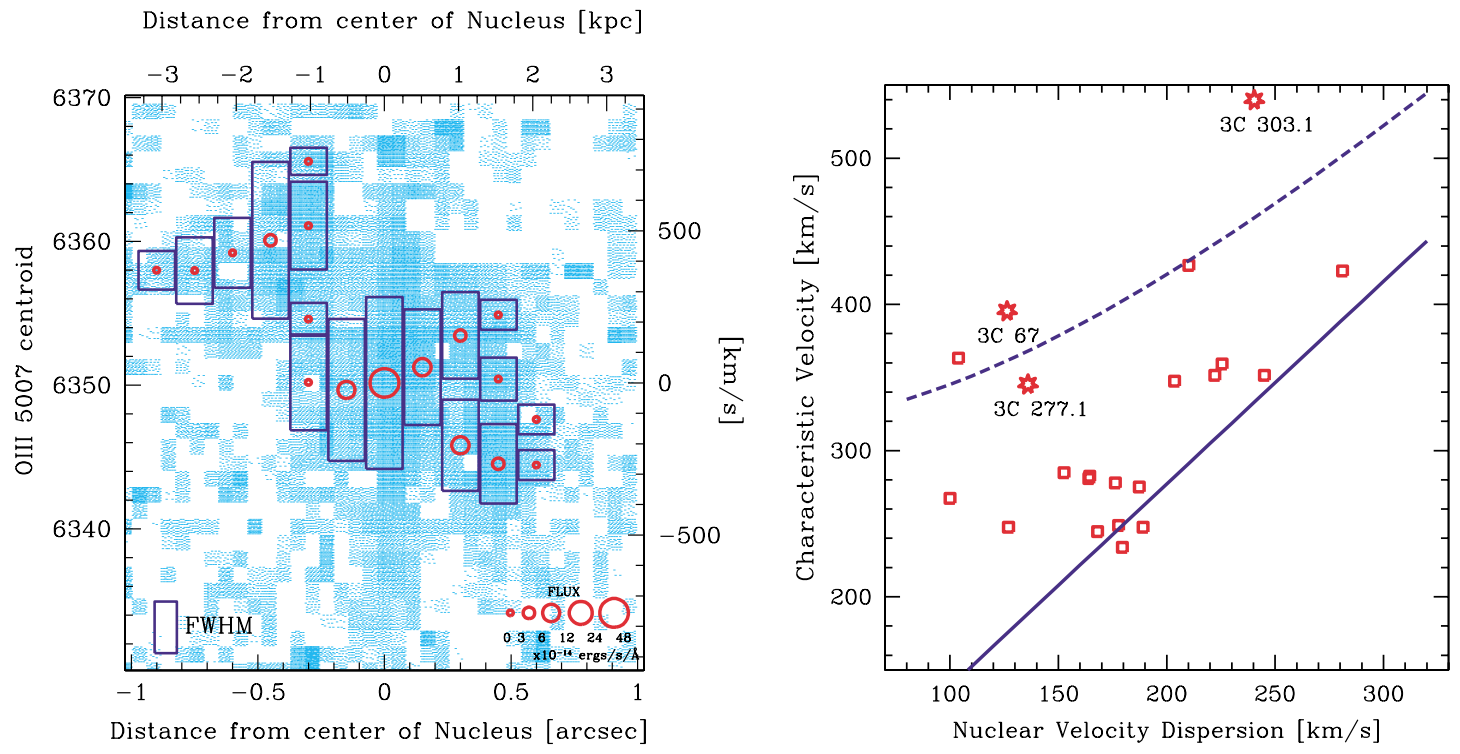

Figure 5 Left: Results of emission-line fitting superposed on a blue-scale plot of the two-dimensional spectrum. Each box shows the location and FWHM of a particular [O III] $5007 \AA ̊$ component, illustrating the resolved and complex velocity structure of 3C 303.1. Right: Our sources (indicated by open stars) compared to nearby comparably powerful radio ellipticals (data from Baum et al. 1990). The solid line represents the non-rotating, laminar flow case. A bipolar outflow, as the most likely operating scenario in our sources, will result in a roughly parallel offset from this line (indicated by the dashed line; both plots are taken from O'Dea et al. (2002).

ratios, strengths, and spatial distributions all can be used to distinguish between, for instance, scattered nuclear light, light due to young stars, or shock induced line emission as the dominant mechanism in the radio aligned emission component. We used HSTs STIS instrument to obtain spatially resolved spectral information on three CSS galaxies (O'Dea et al. 2002). Unlike groundbased spectroscopy, where the slit typically engulfs most of the host galaxy ${ }^{2}$ and therefore presents a blend of properties, the STIS data can be used to isolate the individual components (cf. Figure 5). Furthermore, combining the data over the spatial domain yields high signal-to-noise information that can be compared directly to similar groundbased observations on a local sample of ellipticals (Baum, Heckman, \& van Breugel 1990). Results (see also O’Dea et al. 2003) based on the detailed spectroscopic data indicate that these sources exhibit gas motions consistent with them being driven by the expansion of the radio source. This augments the LRF imaging data in the sense that it most likely rules out a scattered nuclear light scenario for the aligned component.

\section{Emerging Picture}

Based on the information presented here (and more from the literature, cf. O'Dea 1998), a consistent picture seems to emerge for these radio galaxies (Figure 6). GPS, CSS, and FR II radio sources appear to inhabit similar host galaxies. Their hosts display similar colours, have similar inferred formation redshifts/ages, and about the same luminous mass. Profile analysis indicates these sources to have undergone less major mergers (unlike the FR I hosts), and to be more consistent with giant ellipticals, and not

\footnotetext{
${ }^{2}$ Typical galaxy sizes are a few arcseconds at $z \sim 0.3$.
}

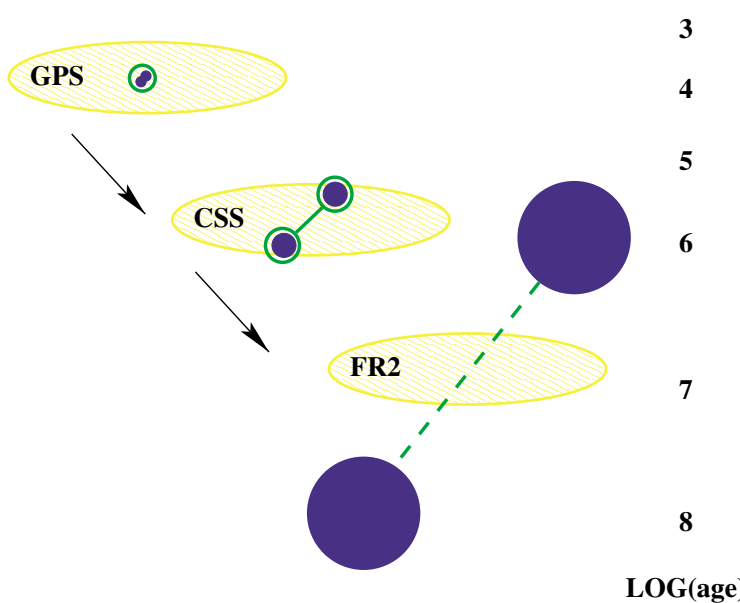

Figure 6 Simple schematic of radio source evolution along the GPS-CSS-FR II sequence. The yellow ellipse is the host galaxy, the blue spheres are the radio lobes, and the green indicates the aligned emission component. In GPS/CSS sources this is most likely shocked line emission gas along the radio structure. In FR II sources the aligned component has to be of a longer lived nature, e.g. scattered nuclear light or star formation.

BCGs (e.g. M87). In short, based on their host galaxies, there is no reason why a GPS source cannot evolve into an FR II. Now, if one assumes GPS/CSS sources evolve into FR IIs, one expects to find supporting evidence, especially in the earlier stages of evolution where the interaction between the powerful jets and the ambient medium is largest. This is exactly what we see in our LRF images and STIS spectroscopy. The narrowband images clearly show emission line gas alongside the (expanding) radio structure. The STIS data confirm that their motion is consistent with being shocked and possibly entrained by the 
jet. Does this mean we are done? No. Even though all the optical/near IR imaging and spectroscopy data point towards the evolutionary picture, some issues remain. First of all, the relative number densities of the radio sources are not consistent with the evolutionary scenario, if one assumes no radio luminosity evolution. This was one of the key arguments in proposing the frustration model for GPS: they are overrepresented by at least 2 orders of magnitude. This apparent paradox can be resolved by assuming the radio sources decline significantly in radio luminosity as they grow (e.g. O’Dea \& Baum 1997) or perhaps even increase in luminosity in the GPS phase before fading in the CSS and FR II phases (Snellen et al. 2000). Both of these scenarios comfortably explain the GPS overdensities. Another potential snag in the simple picture is posed by the GPS quasars. Assuming quasars are just like galaxies, but viewed under a preferred angle, no significantly different properties should be found. This is, however, not the case. Aside from the quite distinct redshift distributions for GPS/CSS galaxies and quasars which barely overlap (e.g. de Vries, Barthel, \& O’Dea 1997; Snellen et al. 1999), $\mathrm{X}$-ray properties have been found to be different as well (O'Dea et al. 2000; Siemiginowska et al. these proceedings). Unless large obscuring (possibly 'frustrating') gas columns render these objects viewing-angle dependent, these sources are different in the sense that the quasars are far more luminous than the galaxies in X-rays. It may just be a selection effect: GPS/CSS quasars peak at intrinsically much higher radio frequencies due to their differing redshift distribution, so we may be comparing apples and oranges. Currently this is, however, too close to call.

\section{Acknowledgments}

WDV's work was performed under the auspices of the US Department of Energy, National Nuclear Security Administration by the University of California, Lawrence Livermore National Laboratory, under contract No. W-7405-Eng-48.

\section{References}

Aragón-Salamanca, A., Ellis, R. S., Couch, W. J., \& Carter D. 1993, MNRAS, 262, 764

Baum, S. A., Heckman, T. M., \& van Breugel, W. 1990, ApJS, 74 389

Bicknell, G., Dopita, M. A., \& O’Dea, C. P. 1997, ApJ, 485, 112

Caon, N., Capaccioli, M., \& D'Onofrio, M. 1993, MNRAS, 265, 1013

Collins, C. A., \& Mann, R. G. 1998, MNRAS, 297, 128

De Breuck, C., van Breugel, W., Stanford, S. A., Röttgering, H., Miley, G., \& Stern, D. 2002, AJ, 123, 637

de Vries, W. H., Barthel, P. D., \& O'Dea, C. P. 1997, A\&A, 321, 105

de Vries, W. H., O’Dea, C. P., Barthel, P. D., \& Thompson, D. J. 2000a, A\&AS, 143, 181

de Vries, W. H., O'Dea, C. P., Baum, S. A., \& Barthel, P. D. 1999, ApJ, 526, 27

de Vries, W. H., O’Dea, C. P., Barthel, P. D., Fanti, C., Fanti, R., \& Lehnert, M. D. 2000b, AJ, 120, 2300

Dey, A., Cimatti, A., van Breugel, W., Antonucci, R., \& Spinrad, H. 1996, ApJ, 465, 157

Graham, A., Lauer, T. R., Colless, M., \& Postman, M. 1996, ApJ, 465, 534

McCarthy, P. J., van Breugel, W. J. M., Spinrad, H., \& Djorgovski, S. 1987, ApJL, 321, L29

O'Dea, C. P. 1998, PASP, 110, 493

O'Dea, C. P., \& Baum, S.A. 1997, AJ, 113, 148

O’Dea, C. P., Stanghellini, C., Baum, S. A., \& Charlot, S. 1996, ApJ, 470,806

O’Dea, C. P., de Vries, W. H., Worrall, D. M., Baum, S. A., \& Koekemoer, A. 2000, AJ, 119, 478

O'Dea, C. P., et al. 2002, AJ, 123, 2333

O'Dea, C. P., et al. 2003, PASA, 20, in press

Owen, F. N., \& Laing, R. A. 1989, MNRAS, 238, 357

Owen, F. N., \& White, R. A. 1991, MNRAS, 249, 164

Rees, M. J. 1989, MNRAS, 239, 1p

Snellen, I. A. G., Bremer, M. N., Schilizzi, R. T., \& Miley, G. K. 1996, MNRAS, 283, L123

Snellen, I. A. G., Schilizzi, R. T., Bremer, M. N., Miley, G. K., de Bruyn, A. G., \& Röttgering, H. J. A. 1999, MNRAS, 307, 149

Snellen, I. A. G., Schilizzi, R. T., Miley, G. K., de Bruyn, A. G., Bremer, M. N., \& Röttgering, H. J. A. 2000, MNRAS, 319, 445 